\title{
A New Finite Element Method for Darcy-Stokes-Brinkman Equations
}

\author{
Bishnu P. Lamichhane \\ School of Mathematical \& Physical Sciences, Mathematics Building, University of Newcastle, \\ University Drive, Callaghan, NSW 2308, Australia \\ Correspondence should be addressed to Bishnu P. Lamichhane; blamichha@gmail.com
}

Received 19 August 2013; Accepted 15 September 2013

Academic Editors: R. K. Upadhyay and W. G. Weng

Copyright (c) 2013 Bishnu P. Lamichhane. This is an open access article distributed under the Creative Commons Attribution License, which permits unrestricted use, distribution, and reproduction in any medium, provided the original work is properly cited.

We present a new finite element method for Darcy-Stokes-Brinkman equations using primal and dual meshes for the velocity and the pressure, respectively. Using an orthogonal basis for the discrete space for the pressure, we use an efficiently computable stabilization to obtain a uniform convergence of the finite element approximation for both limiting cases.

\section{Introduction}

Recently developing an efficient finite element method for Darcy-Stokes-Brinkman problem has become an active area of research [1-5]. This mathematical model is often called a generalized Stokes problem [6] and is also a special case of Oseen's equations when the convective term is absent [1]. This model incorporates both Darcy and Stokes problem. If the viscosity vanishes, the model becomes the Darcy problem, and if another parameter is set to zero, it reduces to Stokes problem. Therefore, this is important when studying the coupling between Stokes and Darcy equations. Some applications of Darcy-Stokes-Brinkman problem are given in [4]. This model also arises from the time discretization of time-dependent Stokes equations.

In this paper we propose a new finite element method for this problem using stabilization based on a local projection. Our technique is the combination of the local projection stabilization for Stokes equations [7] and the grad-div stabilization proposed earlier for the Stokes equations [8]. We note that we mainly focus on the Darcy-Stokes-Brinkman model so that the divergence of the velocity field is not identically zero but is given as a function in $L^{2}$-space as in $[3,4]$. Using a standard continuous linear, bilinear, or trilinear finite element space for the velocity, the grad-div stabilization [8] is to be modified in this case. Since the modification involves projecting the divergence of the velocity field onto the finite element space for the pressure, we first use a discontinuous pressure space in the finite element scheme. This leads to an efficient realization of the stabilization. The finite element space for the pressure is the piecewise constant function space on the dual mesh. The inf-sup condition is satisfied using an element-wise defined bubble function in each element.

This paper is organized as follows. We present the boundary value problem for the Darcy-Stokes-Brinkman problem in the next section. We present our finite element method, propose stabilization, and analyze the discrete problem in Section 3. We prove an optimal a priori error estimate for both cases in this section. Finally, a conclusion is drawn in the last section.

\section{Darcy-Stokes-Brinkman Problem}

This section is devoted to the introduction of the boundary value problem of the Darcy-Stokes-Brinkman flow. Let $\Omega$ in $\mathbb{R}^{d}, d \in\{2,3\}$, be a bounded domain with polygonal or polyhedral boundary $\Gamma$. For a prescribed body force $\mathbf{f} \in$ $\left[L^{2}(\Omega)\right]^{d}$ and another function $g \in L^{2}(\Omega)$, the Darcy-StokesBrinkman equations with homogeneous Dirichlet boundary condition in $\Gamma$ read

$$
\begin{gathered}
-v \Delta \mathbf{u}+\nabla p+\sigma \mathbf{u}=\mathbf{f} \quad \text { in } \Omega, \\
\operatorname{div} \mathbf{u}=g \quad \text { in } \Omega,
\end{gathered}
$$


with $\mathbf{u}=0$ on $\Gamma$, where $\mathbf{u}$ is the velocity, $p$ is the pressure, and $v$ denotes the viscosity of the fluid. For simplicity we assume that $\nu \geq 0$ and $\sigma$ are constant. However, the theory also can be easily extended to cover the case when $\nu$ and $\sigma$ depend on the spatial coordinates $\mathbf{x} \in \Omega$. When the viscosity of the fluid $\nu$ tends to zero, this problem reduces to the Darcy problem, and when the parameter $\sigma$ approaches zero, this problem reduces to the Stokes problem. Here we aim at developing a finite element method which is stable in both limit cases.

Here we use standard notations $L^{2}(\Omega), H^{1}(\Omega)$, and $H_{0}^{1}(\Omega)$ for Sobolev spaces; see $[9,10]$ for details. Let $\mathbf{W}:=\left[H_{0}^{1}(\Omega)\right]^{d}$ be the vector Sobolev space with inner product $(\cdot, \cdot)_{1, \Omega}$ and norm $\|\cdot\|_{1, \Omega}$ defined in the standard way: $(\mathbf{u}, \mathbf{v})_{1, \Omega}:=$ $\sum_{i=1}^{d}\left(u_{i}, v_{i}\right)_{1, \Omega}$, with the norm being induced by this inner product. We also define another subspace $M$ of $L^{2}(\Omega)$ as

$$
M=\left\{q \in L^{2}(\Omega): \int_{\Omega} q d x=0\right\} .
$$

The weak formulation of the Darcy-Stokes-Brinkman problem is to find $(\mathbf{u}, p) \in \mathbf{W} \times M$ such that

$$
\begin{gathered}
v \int_{\Omega} \nabla \mathbf{u}: \nabla \mathbf{v} d x+\int_{\Omega} \operatorname{div} v p d x+\sigma \int_{\Omega} \mathbf{u v} d x=\ell(\mathbf{v}) \\
\mathbf{v} \in \mathbf{W} \\
\int_{\Omega} \operatorname{div} \mathbf{u} q d x=g(q), \quad q \in M,
\end{gathered}
$$

where $\ell(\mathbf{v})=\int_{\Omega} \mathbf{f} \cdot \mathbf{v} d x$. It is well known that the weak formulation of this Darcy-Stokes-Brinkman problem is wellposed $[3,11]$.

We note that, when the viscosity $v \rightarrow 0$, the appropriate space to seek a solution of the variational problem (8) is $H_{0}(\operatorname{div}, \Omega)$ defined as

$$
H_{0}(\operatorname{div}, \Omega)=\{\boldsymbol{\sigma} \in H(\operatorname{div}, \Omega): \boldsymbol{\sigma} \cdot \mathbf{n}=0 \text { on } \partial \Omega\},
$$

where

$$
H(\operatorname{div}, \Omega)=\left\{\mathbf{u} \in\left[L^{2}(\Omega)\right]^{d}: \operatorname{div} \mathbf{u} \in L^{2}(\Omega)\right\}
$$

(see [3]). A standard norm on the space $H(\operatorname{div}, \Omega)$ is

$$
\|\mathbf{u}\|_{\operatorname{div}, \Omega}^{2}=\|\mathbf{u}\|_{0, \Omega}^{2}+\|\operatorname{div} \mathbf{u}\|_{0, \Omega}^{2} .
$$

With this change of the character of the solution, when the viscosity $v$ vanishes, we define an appropriate norm for the problem (8) as

$$
\|\mathbf{v}\|_{a}^{2}:=\nu\|\mathbf{v}\|_{1, \Omega}^{2}+\sigma\|\mathbf{v}\|_{\operatorname{div}, \Omega}^{2}, \quad \mathbf{v} \in[\nu \mathbf{W} \cap \sigma H(\operatorname{div}, \Omega)] .
$$

Therefore now we use the space $\mathbf{V}:=\nu \mathbf{W} \cap \sigma H(\operatorname{div}, \Omega)$ as the space for the velocity $\mathbf{u}$. Due to the occurrences of the parameters in the problem it is appropriate to use the suitable norm defined above although it is clear that
$H(\operatorname{div}, \Omega) \subset\left[H^{1}(\Omega)\right]^{d}$. Thus we consider the problem of finding $(\mathbf{u}, p) \in \mathbf{V} \times M$ such that

$$
\begin{gathered}
v \int_{\Omega} \nabla \mathbf{u}: \nabla \mathbf{v} d x+\int_{\Omega} \operatorname{div} v p d x+\sigma \int_{\Omega} \mathbf{u v} d x=\ell(\mathbf{v}), \\
\mathbf{v} \in \mathbf{W} \\
\int_{\Omega} \operatorname{div} \mathbf{u} q d x=g(q), \quad q \in M .
\end{gathered}
$$

\section{Finite Element Discretizations}

We consider a quasiuniform triangulation $\mathscr{T}_{h}$ of the polygonal or polyhedral domain $\Omega$, where $\mathscr{T}_{h}$ consists of simplices (triangles or tetrahedra), where $h$ denotes the mesh-size. Note that $\mathscr{T}_{h}$ denotes the set of elements.

For nonnegative integer $n$ and an element $K \in \mathscr{T}_{h}$, we let $\mathscr{P}_{n}(K)$ denote the space of polynomials in two variables of total degree less than or equal to $n$, where $K$ is a triangle or tetrahedron. Let $\widehat{K}$ be a reference triangle or tetrahedron, where the reference triangle is defined as

$$
\widehat{K}:=\left\{(x, y) \in \mathbb{R}^{2}: x>0, y>0, x+y<1\right\},
$$

and the reference tetrahedron is

$$
\widehat{K}:=\left\{(x, y, z) \in \mathbb{R}^{3}: x>0, y>0, z>0, x+y+z<1\right\} .
$$

A typical element $K \in \mathscr{T}_{h}$ is generated by an affine map $F_{K}$ from the reference element $\widehat{K}$, in which $F_{K}$ is defined using the basis functions corresponding to $\mathscr{P}_{1}$.

The finite element space of displacement is taken to be the space of continuous functions whose restrictions to an element $K$ are obtained by maps of linear functions from the reference element:

$$
\begin{gathered}
S_{h}:=\left\{v_{h} \in H_{0}^{1}(\Omega), v_{h} \mid K=\widehat{v}_{h} \circ F_{K}^{-1},\right. \\
\left.\widehat{\mathbf{v}}_{h} \in \mathscr{P}_{1}(\widehat{K}), K \in \mathscr{T}_{h}\right\} .
\end{gathered}
$$

Let the set of all vertices in $\mathscr{T}_{h}$ be denoted by $\mathscr{V}_{h}:=\left\{\mathbf{x}_{i}\right\}_{i=1}^{N}$ and $\mathcal{N}_{h}:=\{1,2, \ldots, N\}$.

A dual mesh $\mathscr{T}_{h}^{*}$ is introduced based on the primal mesh $\mathscr{T}_{h}$ so that the elements of $\mathscr{T}_{h}^{*}$ are called control volumes. Each control volume element $V_{i} \in \mathscr{T}_{h}^{*}$ is associated with a vertex $\mathbf{x}_{i} \in \mathscr{V}_{h}$. For simplicity we explain the construction of the dual mesh for triangular meshes $[12,13]$. The construction is easily extended to a tetrahedral mesh. For a vertex $\mathbf{x}_{i} \in \mathscr{V}_{h}$ let $\mathscr{T}_{h}^{i}$ and $\mathscr{E}_{h}^{i}$ be the set of elements and edges touching $\mathbf{x}_{i}$, respectively. Then the volume element $V_{i}$ corresponding to the vertex $\mathbf{x}_{i} \in \mathscr{V}_{h}$ is the polygonal domain joining centroids of all elements in $\mathscr{T}_{h}^{i}$ and centroids of all edges in $\mathscr{E}_{h}^{i}$. The set of all volume elements in $\mathscr{T}_{h}^{*}$ will form a nonoverlapping decomposition of the polygonal domain $\Omega$; see also $[12,13]$. A dual mesh for a triangular mesh is shown in Figure 1.

In the following we use a generic constant $C$, which takes different values at different places but is always independent of the mesh-size $h$ and the parameters $\nu$ and $\sigma$. 


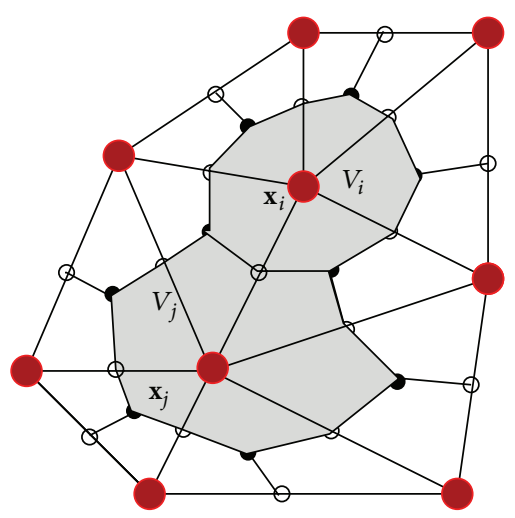

FIgURE 1: Primal and dual meshes for a triangular grid.

We call the control volume mesh $\mathscr{T}_{h}^{*}$ regular or quasiuniform if there exists a positive constant $C>0$ such that

$$
C h^{d} \leq\left|V_{i}\right| \leq h^{d}, \quad V_{i} \in \mathscr{T}_{h}^{*},
$$

where $h$ is the maximum diameter of all elements $T \in \mathscr{T}_{h}$. It can be shown that, if $\mathscr{T}_{h}$ is locally regular, that is, there is a constant $C$ such that

$$
C h_{T}^{d} \leq|T| \leq h_{T}^{d}, \quad T \in \mathscr{T}_{h}
$$

with $\operatorname{diam}(T)=h_{T}$ for all elements $T \in \mathscr{T}_{h}$, then this dual mesh $\mathscr{T}_{h}^{*}$ is also locally regular. The dual volume element space $S_{h}^{*}$ to discretize the pressure is now defined by

$$
S_{h}^{*}:=\left\{p \in L_{0}^{2}(\Omega): p_{\mid V} \in \mathscr{P}_{0}(V), V \in \mathscr{T}_{h}^{*}\right\} .
$$

Now $p_{h} \in S_{h}^{*}$ and $u_{h} \in S_{h}$ can be written as $u_{h}=$ $\sum_{i=1}^{N} u_{i} \phi_{i}$ and $p_{h}=\sum_{i=1}^{N} p_{i} \chi_{i}$, where $\phi_{i}$ are the standard nodal basis functions associated with the vertex $\mathbf{x}_{i}$, and $\chi_{i}$ are the characteristic functions of the volume $V_{i}$.

For the reference element $\widehat{K}$, we define a bubble function

$$
b_{\widehat{K}}
$$$$
= \begin{cases}27 x y(1-x-y), & \text { if } \widehat{K} \text { is the reference triangle, } \\ 256 x y z(1-x-y-z), & \text { if } \widehat{K} \text { is the reference tetrahedron. }\end{cases}
$$

Then defining the space of bubble functions $B_{h}$ as

$$
\begin{aligned}
B_{h} & :=\left\{b_{h} \in H_{0}^{1}(\Omega),\left.b_{h}\right|_{k}\right. \\
& \left.=b_{\widehat{K}} \circ F_{K}^{-1}, b_{\widehat{K}} \text { is a bubble function on } \widehat{K}, K \in \mathscr{T}_{h}\right\},
\end{aligned}
$$

we introduce our finite element space for velocity as $\mathbf{V}_{h}=$ $\left[S_{h} \oplus B_{h}\right]^{d}$.

With this definition of the spaces $\mathbf{V}_{h}$ and $S_{h}^{*}$ we have the following lemma [13].
Lemma 1. There exists a constant $\beta>0$ independent of the mesh-size h such that

$$
\inf _{q_{h} \in S_{h}^{*} \backslash\{0\}} \sup _{\mathbf{v}_{h} \in \mathbf{V}_{h} \backslash\{0\}} \frac{\int_{\Omega} \operatorname{div} \mathbf{v}_{h} q_{h}}{\left\|\mathbf{v}_{h}\right\|_{1, \Omega}\left\|q_{h}\right\|_{0, \Omega}} \geq \beta .
$$

We now consider the limiting case when $v \rightarrow 0$. The main difficulty in this case using the conforming approximation is to get the coercivity of the bilinear form

$$
\widetilde{a}\left(\mathbf{u}_{h}, \mathbf{v}_{h}\right)=\sigma \int_{\Omega} \mathbf{u}_{h} \cdot \mathbf{v}_{h} d x
$$

on the kernel space

$$
\mathbf{K}_{h}:=\left\{\mathbf{v}_{h} \in \mathbf{V}_{h}: \int_{\Omega} \operatorname{div} \mathbf{v}_{h} q_{h} d x=0, q_{h} \in S_{h}^{*}\right\} .
$$

When $v \rightarrow 0$, the coercivity of the bilinear form $\widetilde{a}(\cdot, \cdot)$ on $\mathbf{K}_{h}$ demands the existence of a constant $C>0$ such that

$$
\widetilde{a}\left(\mathbf{v}_{h}, \mathbf{v}_{h}\right) \geq C\left\|\mathbf{v}_{h}\right\|_{a}^{2}=C \sigma\left\|\mathbf{v}_{h}\right\|_{\operatorname{div}, \Omega}^{2} .
$$

Assuming that $\sigma>0$ this condition requires the existence of a constant $C>0$ such that

$$
\left\|\mathbf{v}_{h}\right\|_{0, \Omega} \leq C\left\|\operatorname{div} \mathbf{v}_{h}\right\|_{0, \Omega} .
$$

Since this condition is not satisfied for the space $\mathbf{V}_{h}$, we add a stabilising term to the bilinear form $\widetilde{a}(\cdot, \cdot)$ to obtain another bilinear form $a(\cdot, \cdot)$

$$
\begin{aligned}
a\left(\mathbf{u}_{h}, \mathbf{v}_{h}\right):=\widetilde{a}\left(\mathbf{u}_{h}, \mathbf{v}_{h}\right)+\sigma \int_{\Omega} & \left(\operatorname{div} \mathbf{u}_{h}-Q_{h} \operatorname{div} \mathbf{u}_{h}\right) \\
& \times\left(\operatorname{div} \mathbf{v}_{h}-Q_{h} \operatorname{div} \mathbf{v}_{h}\right) d x,
\end{aligned}
$$

where $Q_{h}$ is an orthogonal projection onto the space $S_{h}^{*}$. With this definition of the bilinear form, our discrete problem is to find $\left[\mathbf{u}_{h}, p_{h}\right] \in \mathbf{V}_{h} \times S_{h}^{*}$ such that

$$
\begin{gathered}
a\left(\mathbf{u}_{h}, \mathbf{v}_{h}\right)+b\left(\mathbf{v}_{h}, p_{h}\right)=\ell\left(\mathbf{v}_{h}\right), \quad \mathbf{v}_{h} \in \mathbf{V}_{h}, \\
b\left(\mathbf{u}_{h}, q_{h}\right)=g\left(q_{h}\right), \quad q_{h} \in S_{h}^{*} .
\end{gathered}
$$

Remark 2. Due to the fact that the basis functions for the space $S_{h}^{*}$ are orthogonal with respect to $L^{2}$-inner product, computing the action of the operator $Q_{h}$ is very easy and efficient. Since the basis functions of $S_{h}^{*}$ are piecewise constant, the stabilization is also local. In fact, similar stabilization can be applied using mini type finite element method [14] at least for a simplicial grid, where computation of the operator $Q_{h}$ will be more difficult, expensive, and nonlocal.

Remark 3. If the source term $g$ is identically zero, the stabilization is simplified as $Q_{h} \operatorname{div} \mathbf{v}_{h}=0$. In fact, such stabilization is called grad-div stabilization and was used before for Stokes equations in [8].

We now show that the bilinear form $a(\cdot, \cdot)$ is uniformly coercive on $\mathbf{K}_{h}$ with respect to the norm $\|\cdot\|_{a}$. 
Lemma 4. One has

$$
a\left(\mathbf{v}_{h}, \mathbf{v}_{h}\right)=v\left\|\mathbf{v}_{h}\right\|_{1, \Omega}^{2}+\sigma\left\|\mathbf{v}_{h}\right\|_{\operatorname{div}, \Omega}^{2}, \quad \mathbf{v}_{h} \in \mathbf{K}_{h} .
$$

Proof. Note that

$$
\begin{aligned}
a\left(\mathbf{v}_{h}, \mathbf{v}_{h}\right)= & v\left\|\mathbf{v}_{h}\right\|_{1, \Omega}^{2}+\sigma\left\|\mathbf{v}_{h}\right\|_{0, \Omega}^{2} \\
& +\sigma\left\|\operatorname{div} \mathbf{v}_{h}-Q_{h} \operatorname{div} \mathbf{v}_{h}\right\|_{0, \Omega}^{2} \\
= & v\left\|\mathbf{v}_{h}\right\|_{1, \Omega}^{2}+\sigma\left\|\mathbf{v}_{h}\right\|_{0, \Omega}^{2}+\sigma\left\|\operatorname{div} \mathbf{v}_{h}\right\|_{0, \Omega}^{2} \\
= & v\left\|\mathbf{v}_{h}\right\|_{1, \Omega}^{2}+\sigma\left\|\mathbf{v}_{h}\right\|_{\operatorname{div}, \Omega}^{2},
\end{aligned}
$$

as $Q_{h} \operatorname{div} \mathbf{v}_{h}=0$ for $\mathbf{v}_{h} \in K_{h}$.

Clearly, we see that the bilinear forms $a(\cdot, \cdot)$ and $b(\cdot, \cdot)$, and the linear form $\ell(\cdot)$ are also continuous on the spaces where they are defined. Moreover, since for a constant $C>0$ we have

$$
\left\|\mathbf{v}_{h}\right\|_{a} \leq C\left\|\mathbf{v}_{h}\right\|_{1, \Omega},
$$

we have the inf-sup condition from Lemma 1 when the $\|\cdot\|_{1, \Omega}$ is replaced by $\|\cdot\|_{a}$. Thus we have the following theorem.

Theorem 5. The discrete saddle point problem (23) has a unique solution $\left[\mathbf{u}_{h}, p_{h}\right] \in \mathbf{V}_{h} \times S_{h}^{*}$ such that

$$
\left\|\mathbf{u}_{h}\right\|_{a}+\left\|p_{h}\right\|_{0, \Omega} \leq C\|\ell\|_{0, \Omega} \text {. }
$$

The error estimate is given in the following theorem.

Theorem 6. Let $[\mathbf{u}, p] \in \mathbf{V} \times M$ be the unique solution of (8) and $\left[\mathbf{u}_{h}, p_{h}\right] \in \mathbf{V}_{h} \times S_{h}^{*}$ that of the discrete (23). One has the following error estimate uniformly with respect to $\sigma$ and $v$ :

$$
\begin{aligned}
\| \mathbf{u} & -\mathbf{u}_{h}\left\|_{a}+\right\| p-p_{h} \|_{0, \Omega} \\
& \leq C\left(\inf _{\mathbf{v}_{h} \in \mathbf{V}_{h}}\left\|\mathbf{u}-\mathbf{v}_{h}\right\|_{a}+\inf _{q_{h} \in S_{h}^{*}}\left\|p-q_{h}\right\|_{0, \Omega}\right) .
\end{aligned}
$$

\section{Conclusion}

We have presented a very efficient finite element technique for approximating the solution of Darcy-Stokes-Brinkman equations. Using local projection stabilization for the limiting case of vanishing viscosity we have shown that the error estimate is uniform for both limiting cases.

\section{References}

[1] E. Burman and P. Hansbo, "Stabilized Crouzeix-Raviart element for the Darcy-Stokes problem," Numerical Methods for Partial Differential Equations, vol. 21, no. 5, pp. 986-997, 2005.

[2] H. Rui and R. Zhang, "A unified stabilized mixed finite element method for coupling Stokes and Darcy flows," Computer Methods in Applied Mechanics and Engineering, vol. 198, no. 33-36, pp. 2692-2699, 2009.

[3] K. A. Mardal, X.-C. Tai, and R. Winther, "A robust finite element method for Darcy-Stokes flow," SIAM Journal on Numerical Analysis, vol. 40, no. 5, pp. 1605-1631, 2002.
[4] X. Xie, J. Xu, and G. Xue, "Uniformly-stable finite element methods for Darcy-Stokes-Brinkman models," Journal of Computational Mathematics, vol. 26, no. 3, pp. 437-455, 2008.

[5] T. Karper, K.-A. Mardal, and R. Winther, "Unified finite element discretizations of coupled darcy-stokes flow," Numerical Methods for Partial Differential Equations, vol. 25, no. 2, pp. 311-326, 2009.

[6] S. H. Chou, "Analysis and convergence of a covolume method for the generalized stokes problem," Mathematics of Computation, vol. 66, no. 217, pp. 85-104, 1997.

[7] P. B. Bochev, C. R. Dohrmann, and M. D. Gunzburger, "Stabilization of low-order mixed finite elements for the stokes equations," SIAM Journal on Numerical Analysis, vol. 44, no. 1, pp. 82-101, 2006.

[8] M. A. Olshanskii and A. Reusken, "Grad-Div stablilization for stokes equations," Mathematics of Computation, vol. 73, no. 248, pp. 1699-1718, 2004.

[9] S. C. Brenner and L. R. Scott, The Mathematical Theory of Finite Element Methods, Springer, New York, NY, USA, 1994.

[10] P. G. Ciarlet, The Finite Element Method for Elliptic Problems, North-Holland, Amsterdam, The Netherlands, 1978.

[11] E. Burman and P. Hansbo, "A stabilized non-conforming finite element method for incompressible flow," Computer Methods in Applied Mechanics and Engineering, vol. 195, no. 23-24, pp. 28812899, 2006.

[12] R. E. Ewing, T. Lin, and Y. Lin, "On the accuracy of the finite volume element method based on piecewise linear polynomials," SIAM Journal on Numerical Analysis, vol. 39, no. 6, pp. 18651888, 2002.

[13] B. P. Lamichhane, "Inf-sup stable finite-element pairs based on dual meshes and bases for nearly incompressible elasticity," IMA Journal of Numerical Analysis, vol. 29, no. 2, pp. 404-420, 2009.

[14] D. N. Arnold, F. Brezzi, and M. Fortin, "A stable finite element for the stokes equations," Calcolo, vol. 21, no. 4, pp. 337-344, 1984. 


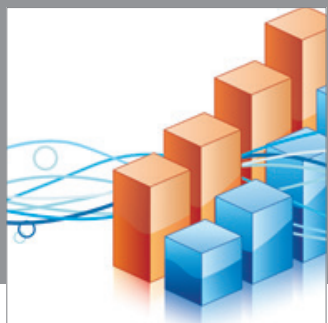

Advances in

Operations Research

mansans

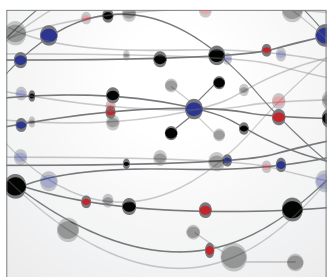

The Scientific World Journal
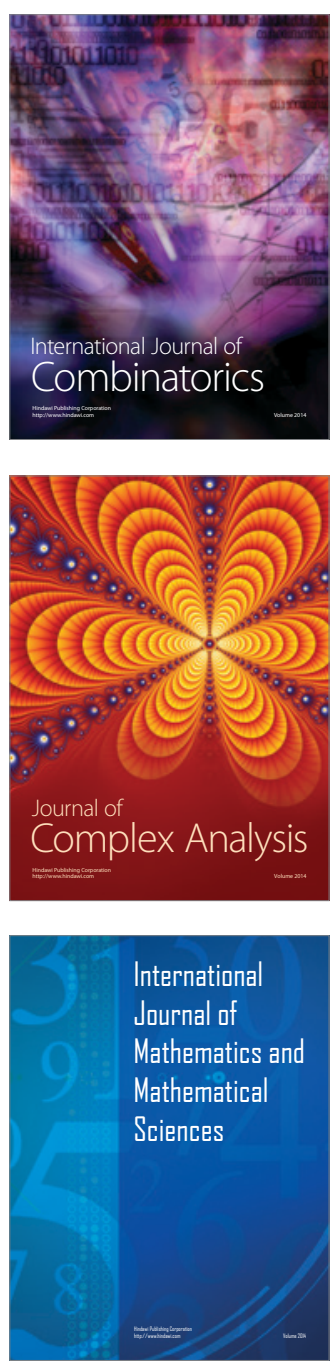
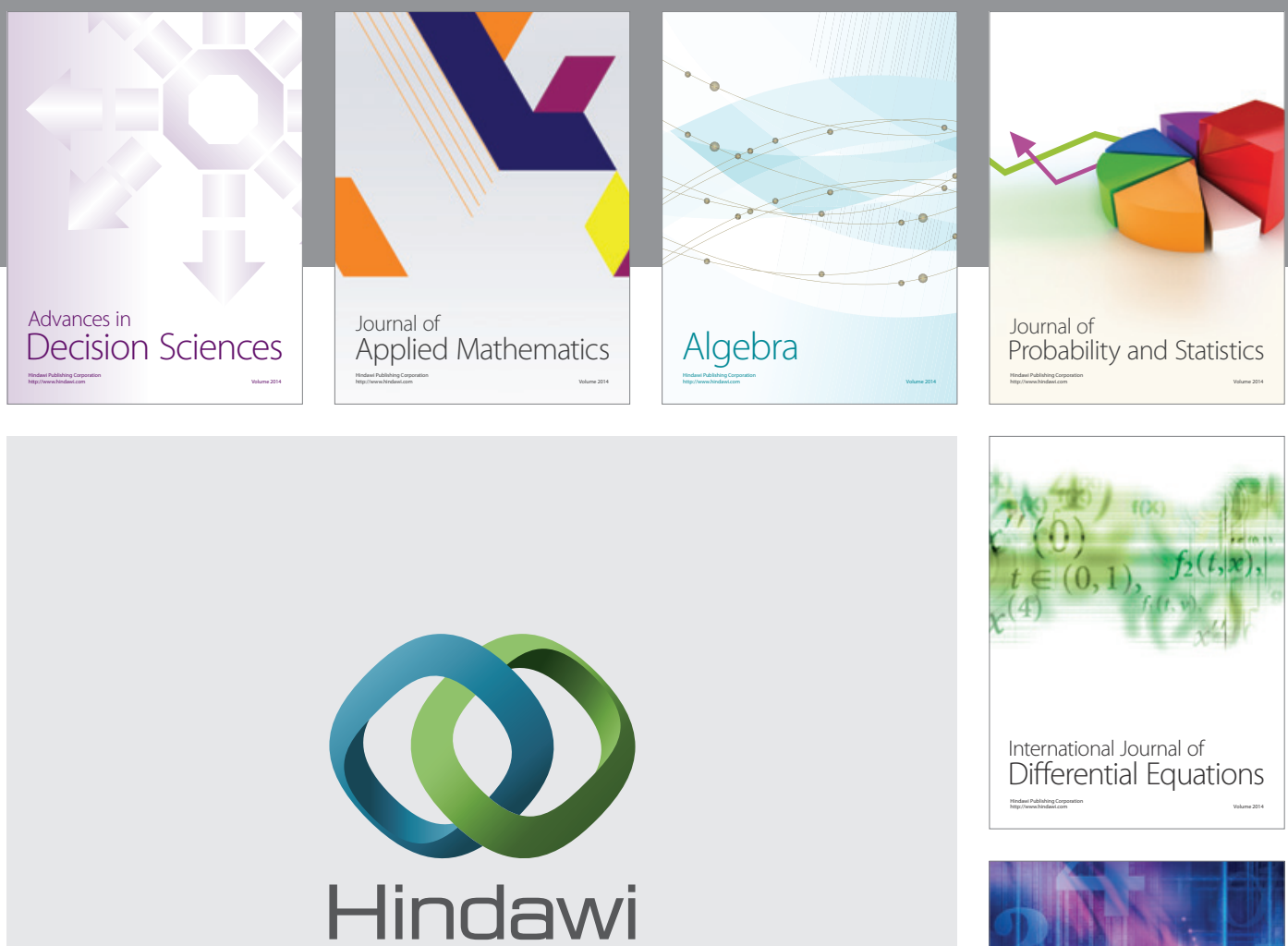

Submit your manuscripts at http://www.hindawi.com
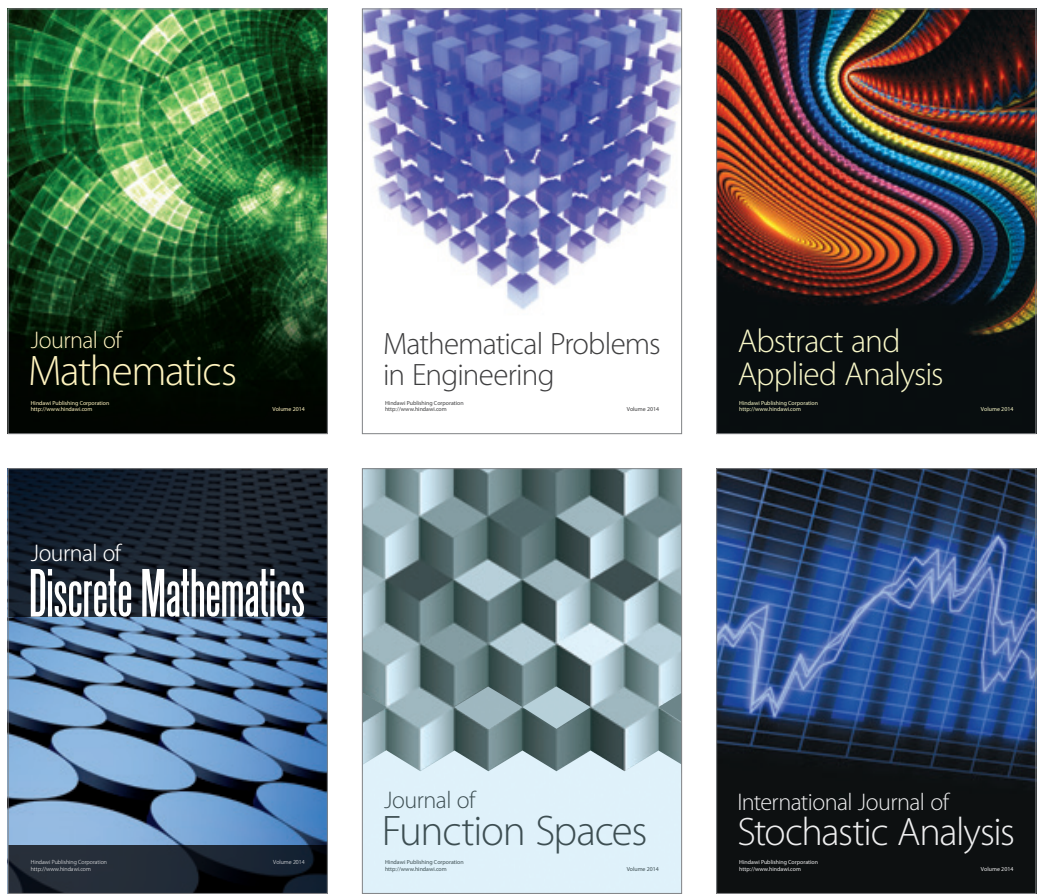

Journal of

Function Spaces

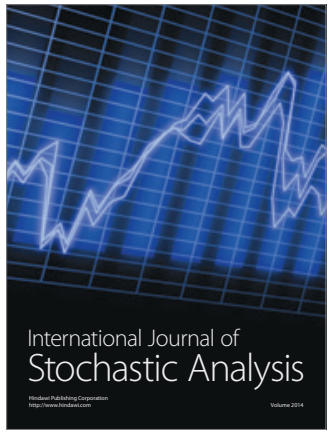

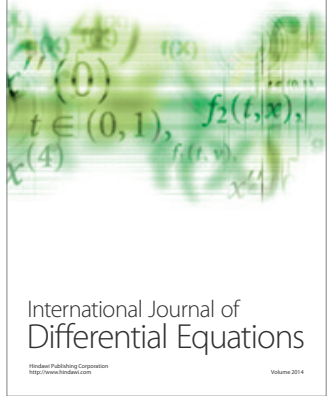
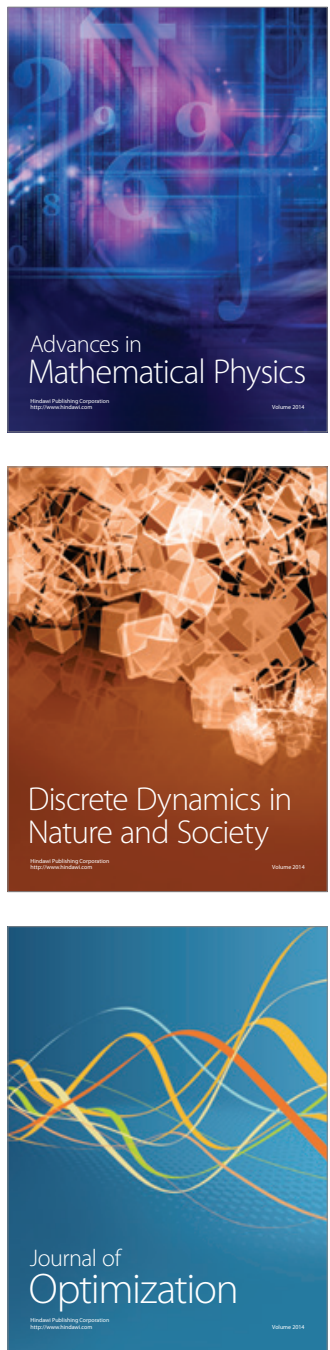